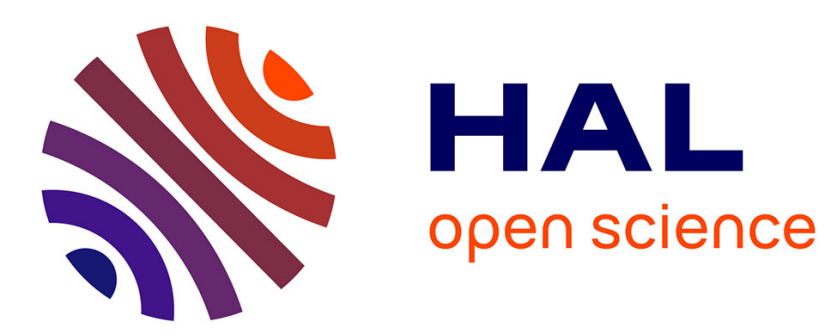

\title{
A SIMPLE PROOF OF A MULTIDIMENSIONAL BORG-LEVINSON TYPE THEOREM
}

\author{
Mourad Choulli
}

\section{To cite this version:}

Mourad Choulli. A SIMPLE PROOF OF A MULTIDIMENSIONAL BORG-LEVINSON TYPE THEOREM. 2019. hal-02406382

\section{HAL Id: hal-02406382 \\ https://hal.science/hal-02406382}

Preprint submitted on 12 Dec 2019

HAL is a multi-disciplinary open access archive for the deposit and dissemination of scientific research documents, whether they are published or not. The documents may come from teaching and research institutions in France or abroad, or from public or private research centers.
L'archive ouverte pluridisciplinaire HAL, est destinée au dépôt et à la diffusion de documents scientifiques de niveau recherche, publiés ou non, émanant des établissements d'enseignement et de recherche français ou étrangers, des laboratoires publics ou privés. 


\title{
A SIMPLE PROOF OF A MULTIDIMENSIONAL BORG-LEVINSON TYPE THEOREM
}

\author{
MOURAD CHOULLI
}

\begin{abstract}
We provide a simple and short proof of a multidimensional BorgLevinson type theorem. Precisely, we prove that the spectral boundary data determine uniquely the corresponding potential appearing in the Schödinger operator on an admissible Riemannian manifold. We also sketch the proof of the case of incomplete spectral boundary data.
\end{abstract}

\section{INTRODUCTION}

Let $\mathcal{M}=(\mathcal{M}, \mathfrak{g})$ be a smooth compact Riemannian manifold of dimension $n \geq 3$ and with boundary $\partial \mathcal{M}$. We recall that, in local coordinates, the usual LaplaceBeltrami operator is given by

$$
\Delta_{\mathfrak{g}}=\frac{1}{\sqrt{|\mathfrak{g}|}} \sum_{j, k=1}^{n} \frac{\partial}{\partial x_{j}}\left(\sqrt{|\mathfrak{g}|} \mathfrak{g}^{j k} \frac{\partial}{\partial x_{k}} \cdot\right) .
$$

We define, for $q \in L^{\infty}(\mathcal{M}, \mathbb{R})$, the unbounded operator $L=L(q)$ acting on $L^{2}(\mathcal{M})$ as follows

$$
L=-\Delta_{\mathfrak{g}}+q \quad \text { with } \quad D(L)=H_{0}^{1}(\mathcal{M}) \cap H^{2}(\mathcal{M}) .
$$

As $L$ is self-adjoint operator with compact resolvent, its spectrum is reduced to a sequence of eigenvalues:

$$
-\infty<\lambda_{1} \leq \ldots \lambda_{k} \leq \ldots \text { and } \quad \lambda_{k} \rightarrow \infty \text { as } k \rightarrow \infty .
$$

Of course $\lambda_{k}=\lambda_{k}(q), k \geq 1$.

In addition, there exists $\left(\phi_{k}\right), \phi_{k}=\phi_{k}(q), k \geq 1$, an orthonormal basis of $L^{2}(\mathcal{M})$ consisting of eigenfunctions. Each $\phi_{k}$ is associated to $\lambda_{k}, k \geq 1$.

For simplicity convenience, we use the notation

$$
\psi_{k}=\left.\partial_{\nu} \phi_{k}\right|_{\partial \mathcal{M}}, \quad k \geq 1,
$$

where $\nu$ is the unit normal vector field on $\partial \mathcal{M}$ with respect to $\mathfrak{g}$.

When $q \in L_{+}^{\infty}(\mathcal{M})=\left\{q \in L^{\infty}(\mathcal{M}, \mathbb{R}) ; q \geq 0\right\}, 0$ is not in the spectrum of $L$ and all the eigenvalues of $L$ are positive. Then, for $f \in H^{1 / 2}(\partial \mathcal{M})$, the BVP

$$
\left(-\Delta_{\mathfrak{g}}+q\right) u=0 \text { in } \mathcal{M} \text { and } \mathrm{u}=f \text { on } \partial \mathcal{M}
$$

Date: December 12, 2019.

2010 Mathematics Subject Classification. 35R30, 35J10.

Key words and phrases. Admissible Riemannian manifold, boundary spectral data, BorgLevinson type theorem, Dirichlet-to-Neumann map.

The author supported by the grant ANR-17-CE40-0029 of the French National Research Agency ANR (project MultiOnde). 
admits a unique solution $u=u(q, f) \in H^{1}(\mathcal{M})$ with $\partial_{\nu} u \in H^{-1 / 2}(\partial \mathcal{M})$. Moreover

$$
\left\|\partial_{\nu} u\right\|_{H^{-1 / 2}(\partial \mathcal{M})} \leq C\|f\|_{H^{1 / 2}(\partial \mathcal{M})}
$$

where the constant $C$ is independent of $f$. In other words, the mapping

$$
\Lambda(q): f \in H^{1 / 2}(\partial \mathcal{M}) \rightarrow \partial_{\nu} u \in H^{-1 / 2}(\partial \mathcal{M})
$$

defines a bounded operator. The operator $\Lambda(q)$ is usually called the Dirichlet-toNeumann map associated to $q$.

For $\tilde{q} \in L_{+}^{\infty}(\mathcal{M})$, we set $\tilde{\lambda}_{k}=\lambda_{k}(\tilde{q}), \tilde{\phi}_{k}=\phi_{k}(\tilde{q})$ and $\tilde{\psi}_{k}=\psi_{k}(\tilde{q})$. We introduce then the notation

$$
\mathfrak{D}(q, \tilde{q})=\sum_{k \geq 1}\left|\lambda_{k}-\tilde{\lambda}_{k}\right|+\sum_{k \geq 1}\left\|\tilde{\psi}_{k}-\psi_{k}\right\|_{L^{2}(\partial \mathcal{M})} .
$$

We aim to prove the following result:

Theorem 1.1. Let $\aleph>0$. If $0 \leq q \leq \aleph, 0 \leq \tilde{q} \leq \aleph$ and $\mathfrak{D}(q, \tilde{q})<\infty$, then $\Lambda(q)-\Lambda(\tilde{q})$ extends to a bounded operator on $L^{2}(\partial \mathcal{M})$ and

$$
\|\Lambda(q)-\Lambda(\tilde{q})\|_{\mathscr{B}\left(L^{2}(\partial \mathcal{M})\right)} \leq C \mathfrak{D}(q, \tilde{q}),
$$

where the constant $C$ only depends on $n, \mathcal{M}$ and $\aleph$.

Let $(\mathscr{M}, g)$ a compact Riemannian manifold with boundary $\partial \mathscr{M}$. We say that $\mathscr{M}$ is admissible if $\mathscr{M} \Subset \mathbb{R} \times \mathscr{M}_{0}$, for some (n-1)-dimensional simple manifold $\left(\mathscr{M}_{0}, g_{0}\right)$, and if $g=c\left(\mathfrak{e} \oplus g_{0}\right)$, where $\mathfrak{e}$ is the Euclidean metric on $\mathbb{R}$ and $c$ is a smooth positive function on $\mathscr{M}$. We recall that $\left(\mathscr{M}_{0}, g_{0}\right)$ is simple if it is a compact Riemannian manifold with boundary and, for any $x \in \mathscr{M}_{0}$, the exponential map $\exp _{x}$ with its maximal domain of definition is a diffeomorphism onto $\mathscr{M}_{0}$, and $\partial \mathscr{M}_{0}$ is strictly convex (that is, the second fundamental form of $\partial \mathscr{M}_{0} \hookrightarrow \mathscr{M}_{0}$ is positive definite).

Observing that

$$
\|\Lambda(q)-\Lambda(\tilde{q})\|_{\mathscr{B}\left(H^{1 / 2}(\partial \mathcal{M}), H^{-1 / 2}(\partial \mathcal{M})\right)} \leq\|\Lambda(q)-\Lambda(\tilde{q})\|_{\mathscr{B}\left(L^{2}(\partial \mathcal{M})\right)}
$$

and $\mathfrak{D}(q, \tilde{q})=\mathfrak{D}(q-\lambda, \tilde{q}-\lambda)$, for any $\lambda$ not belonging to the spectrum of $L(q)$ and $L(\tilde{q})$, we deduce as a consequence of Theorem 1.1 and $[8$, Theorem 1] the following stability inequality:

Corollary 1.1. Let $\aleph>0, t \in(0,1 / 2)$ and assume that $\mathcal{M}$ is admissible with $\mathcal{M} \Subset \mathbb{R} \times \mathcal{M}_{0}$. Then there exist two constants $C>0$ and $0<c<1$, only depending on $n, \mathcal{M}$ and $\aleph$, so that, for any $q, \tilde{q} \in L^{\infty}(\mathcal{M}, \mathbb{R}) \cap H^{t}(\mathcal{M})$ satisfying

$$
\|q\|_{L^{\infty}(\mathcal{M}) \cap H^{t}(\mathcal{M})} \leq \aleph, \quad\|\tilde{q}\|_{L^{\infty}(\mathcal{M}) \cap H^{t}(\mathcal{M})} \leq \aleph \quad \text { and } \quad \mathfrak{D}(q, \tilde{q}) \leq c,
$$

we have

$$
\|q-\tilde{q}\|_{L^{2}\left(\mathbb{R}, H^{-3}\left(\mathcal{M}_{0}\right)\right)} \leq C\left|\ln \left[\mathfrak{D}(q, \tilde{q})+|\ln (\mathfrak{D}(q, \tilde{q}))|^{-1}\right]\right|^{-t / 4} .
$$

In particular, this corollary says that in an admissible manifold the spectral boundary data $\left(\lambda_{k}(q), \psi_{k}(q)\right)_{k \geq 1}$ determine uniquely $q \in L^{\infty}(\mathcal{M}, \mathbb{R}) \cap H^{t}(\mathcal{M})$ :

Theorem 1.2. Let $t \in(0,1 / 2)$ and assume that $\mathcal{M}$ is admissible. If $q, \tilde{q} \in$ $L^{\infty}(\mathcal{M}, \mathbb{R}) \cap H^{t}(\mathcal{M})$ satisfy

$$
\lambda_{k}(q)=\lambda_{k}(\tilde{q}) \quad \text { and } \quad \psi_{k}(q)=\psi_{k}(\tilde{q}), \quad k \geq 1,
$$

then $q=\tilde{q}$. 
Results of the same kind as Theorem 1.2 are known in the literature as multidimensional Borg-Levinson type theorems.

The paper by Nachman, Sylvester and Uhlmann [18] was the starting point of new developments and results on multidimensional Borg-Levinson type theorems. We quote here the following short list of references: $[1,2,3,4,6,7,9,10,12,13$, $14,15,16,17]$. Of course many other works on spectral inverse problems exist in the literature. A short survey on multidimensional Borg-Levinson type theorems can be found in [4]. The case of unbounded potentials was considered by Pohjola in [19]. We also mention that a new stability inequality for the two dimensional case was recently established by Imanuvilov and Yamamoto in [11].

\section{Proof of the MAIN RESUlT}

Henceforward, the usual scalar products on $L^{2}(\mathcal{M})$ and $L^{2}(\partial \mathcal{M})$ are denoted respectively by $(\cdot \mid \cdot)$ and $\langle\cdot \mid \cdot\rangle$.

From [4, Lemma 3.1], the solution of the BVP 1.1 is given by

$$
u=-\sum_{k \geq 1} \frac{\left\langle\psi_{k} \mid f\right\rangle}{\lambda_{k}} \phi_{k} .
$$

This identity yields

$$
\sum_{k \geq 1} \frac{\left|\left\langle\psi_{k} \mid f\right\rangle\right|^{2}}{\lambda_{k}^{2}}=\|u\|_{L^{2}(\mathcal{M})}^{2}
$$

Pick $\left(\rho_{k}\right)$ an orthonormal basis of $L^{2}(\mathcal{M}), s \geq 0$ and define

$$
\mathscr{H}^{s}=\left\{w \in H^{2}(\mathcal{M}), \sum_{k \geq 1} k^{4 s / n}\left(k^{4 / n}\left|\left(w \mid \rho_{k}\right)\right|^{2}+\left|\left(\Delta_{\mathfrak{g}} w \mid \rho_{k}\right)\right|^{2}\right)<\infty\right\} .
$$

We have by Parseval's identity

$$
\begin{aligned}
\sum_{k \geq 1} k^{4 s / n}\left(k^{4 / n}\left|\left(w \mid \rho_{k}\right)\right|^{2}+\left|\left(\Delta_{\mathfrak{g}} w \mid \rho_{k}\right)\right|^{2}\right) & \\
= & \left\|\sum_{k \geq 1} k^{2(s+1) / n}\left(w \mid \rho_{k}\right) \rho_{k}\right\|_{L^{2}(\mathcal{M})}^{2}+\left\|\sum_{k \geq 1} k^{2 s / n}\left(\Delta_{\mathfrak{g}} w \mid \rho_{k}\right) \rho_{k}\right\|_{L^{2}(\mathcal{M})}^{2} .
\end{aligned}
$$

In consequence, the left hand side of this identity does not depend on the choice of the orthonormal basis $\left(\rho_{k}\right)$. We endow $\mathscr{H}^{s}$ with the norm

$$
\|w\|_{\mathscr{H}^{s}}=\|w\|_{H^{2}(\mathcal{M})}+\left(\sum_{k \geq 1} k^{4 s / n}\left(k^{4 / n}\left|\left(w \mid \rho_{k}\right)\right|^{2}+\left|\left(\Delta_{\mathfrak{g}} w \mid \rho_{k}\right)\right|^{2}\right)\right)^{1 / 2} .
$$

It is not hard check that $\mathscr{H}^{s}$ contains all the eigenfunctions $\left(\varphi_{k}\right)$ of the operator $-\Delta_{\mathfrak{g}}$, under Neumann boundary condition, and therefore it contains also the closure, with respect to $\|\cdot\|_{\mathscr{H}}$, of the vector space spanned by these eigenfunctions. This can be easily seen just by taking $\rho_{k}=\varphi_{k}, k \geq 1$, in the definition of $\mathscr{H}^{s}$.

We associate to $\mathscr{H}^{s}$ the trace space

$$
\mathfrak{H}^{s}=\left\{g \in H^{3 / 2}(\partial \mathcal{M}) ; g=\left.w\right|_{\partial \mathcal{M}} \text { for some } w \in \mathscr{H}^{s}\right\}
$$


that we equip with its natural quotient norm

$$
\|g\|_{\mathfrak{H}^{s}}=\inf \left\{\|w\|_{\mathscr{H}} ;\left.w\right|_{\partial \mathcal{M}}=g\right\} .
$$

Lemma 2.1. We fix $s>n / 4$. Then, for any $g \in \mathfrak{H}^{s}$, the series $\sum_{k \geq 1} \frac{\left\langle\psi_{k} \mid g\right\rangle}{\lambda_{k}} \phi_{k}$ converges in $H^{2}(\mathcal{M})$ with

$$
\sum_{k \geq 1} \frac{\left|\left\langle\psi_{k} \mid g\right\rangle\right|}{\lambda_{k}}\left\|\phi_{k}\right\|_{H^{2}(\mathcal{M})} \leq C\|g\|_{\mathfrak{H}^{s}}
$$

where the constant $C$ is independent of $g$.

Proof. Let $g \in \mathfrak{H}^{s}$ and $w \in \mathscr{H}^{s}$ arbitrary so that $\left.w\right|_{\partial \mathcal{M}}=g$. From Green's Formula, we have

$$
\begin{aligned}
\left\langle\psi_{k} \mid g\right\rangle=\int_{\partial \mathcal{M}} \partial_{\nu} \phi_{k} \bar{g} d \sigma & =\int_{\mathcal{M}} \Delta_{\mathfrak{g}} \phi_{k} \bar{w} d v-\int_{\mathcal{M}} \phi_{k} \overline{\Delta_{\mathfrak{g}} w} d v \\
& =\int_{\mathcal{M}}\left(-\lambda_{k}+q\right) \phi_{k} \bar{w} d v-\int_{\mathcal{M}} \phi_{k} \overline{\Delta_{\mathfrak{g}} w} d v .
\end{aligned}
$$

Therefore, taking into account that $\lambda_{k} \sim k^{2 / n}$ as $k \rightarrow+\infty$ (by Weyl's asymptotic formula), we get

$$
\left|\left\langle\psi_{k} \mid g\right\rangle\right| \leq C\left(k^{2 / n}\left|\left(\phi_{k} \mid w\right)\right|+\left|\left(\phi_{k} \mid \Delta_{\mathfrak{g}} w\right)\right|\right), \quad k \geq 1,
$$

where the constant $C$ is independent of $w$.

But $\left\|\phi_{k}\right\|_{H^{2}(\mathcal{M})} \leq C \lambda_{k}$ for any $k \geq 1$. Hence

$$
\frac{\left|\left\langle\psi_{k} \mid g\right\rangle\right|}{\lambda_{k}}\|\phi\|_{H^{2}(\mathcal{M})} \leq C k^{-2 s / n}\left[k^{2 s / n}\left(k^{2 / n}\left|\left(\phi_{k} \mid w\right)\right|+\left|\left(\phi_{k} \mid \Delta_{\mathfrak{g}} w\right)\right|\right)\right], \quad k \geq 1,
$$

Whence, the series $\sum_{k \geq 1} \frac{\left\langle\psi_{k} \mid g\right\rangle}{\lambda_{k}} \phi_{k}$ converges in $H^{2}(\mathcal{M})$ and using Cauchy-Schwarz's inequality we find

$$
\sum_{k \geq 1} \frac{\left|\left\langle\psi_{k} \mid g\right\rangle\right|}{\lambda_{k}}\left\|\phi_{k}\right\|_{H^{2}(\mathcal{M})} \leq C\|w\|_{\mathscr{H}} s
$$

As $w \in \mathscr{H}^{s}$ is chosen arbitrary so that $\left.w\right|_{\partial \mathcal{M}}=g$, inequality (2.1) follows.

Proof of Theorem 1.1. In this proof $C$ is a generic constant depending only on $n$, $\mathcal{M}$ and $\aleph$.

In light of Lemma 2.1, we have

$$
\Lambda(q)(f)=-\sum_{k \geq 1} \frac{\left\langle\psi_{k} \mid f\right\rangle}{\lambda_{k}} \psi_{k}, \quad f \in \mathfrak{H}^{s}
$$

and

$$
\Lambda(\tilde{q})(f)=-\sum_{k \geq 1} \frac{\left\langle\tilde{\psi}_{k} \mid f\right\rangle}{\tilde{\lambda}_{k}} \tilde{\psi}_{k}, \quad f \in \mathfrak{H}^{s} .
$$

We split $\Lambda_{q}(f)-\Lambda_{\tilde{q}}(f), f \in \mathfrak{H}^{s}$, into three terms:

$$
\Lambda_{q}(f)-\Lambda_{\tilde{q}}(f)=A_{1}+A_{2}+A_{3},
$$


with

$$
\begin{aligned}
& A_{1}=\sum_{k \geq 1}\left(\frac{1}{\tilde{\lambda}_{k}}-\frac{1}{\lambda_{k}}\right)\left\langle\tilde{\psi}_{k} \mid f\right\rangle \tilde{\psi}_{k}, \\
& A_{2}=\sum_{k \geq 1} \frac{\left\langle\tilde{\psi}_{k} \mid f\right\rangle-\left\langle\psi_{k} \mid f\right\rangle}{\lambda_{k}} \tilde{\psi}_{k}, \\
& A_{3}=\sum_{k \geq 1} \frac{\left\langle\psi_{k} \mid f\right\rangle}{\lambda_{k}}\left(\tilde{\psi}_{k}-\psi_{k}\right) .
\end{aligned}
$$

Then it is straightforward to check that

$$
\begin{aligned}
& \left\|A_{1}\right\|_{L^{2}(\partial \mathcal{M})} \leq C \sum_{k \geq 1}\left|\lambda_{k}-\tilde{\lambda}_{k}\right|\|f\|_{L^{2}(\partial \mathcal{M})}, \\
& \left\|A_{2}\right\|_{L^{2}(\partial \mathcal{M})}+\left\|A_{3}\right\|_{L^{2}(\partial \mathcal{M})} \leq C \sum_{k \geq 1}\left\|\tilde{\psi}_{k}-\psi_{k}\right\|_{L^{2}(\partial \mathcal{M})}\|f\|_{L^{2}(\partial \mathcal{M})} .
\end{aligned}
$$

Therefore, under the assumption $\mathfrak{D}(q, \tilde{q})<\infty$, the operator $\Lambda_{q}-\Lambda_{\tilde{q}}$ extends to a bounded operator on $L^{2}(\partial \mathcal{M})$ with

$$
\left\|\Lambda_{q}-\Lambda_{\tilde{q}}\right\|_{\mathscr{B}\left(L^{2}(\partial \mathcal{M})\right)} \leq C \mathfrak{D}(q, \tilde{q})
$$

The proof is then complete.

\section{The CASE OF incomplete SPECTRAL BOUndary DATA}

We explain briefly in this short section how we can get a Borg-Levinson type theorem for incomplete spectral boundary data. For this purpose, we assume that $\mathcal{M}$ is simple. In that case it is known that there exists another simple manifold $\mathcal{M}_{1}$ so that $\mathcal{M}_{1} \ni \mathcal{M}$.

Let $q, \tilde{q} \in L^{\infty}(\mathcal{M}, \mathbb{R})$ satisfying $p=(q-\tilde{q}) \chi_{\mathcal{M}} \in H^{1}\left(\mathcal{M}_{1}\right)$,

$$
\|q\|_{L^{\infty}(\mathcal{M})} \leq \aleph, \quad\|\tilde{q}\|_{L^{\infty}(\mathcal{M})} \leq \aleph \quad \text { and } \quad\|p\|_{H^{1}\left(\mathcal{M}_{1}\right)} \leq \aleph .
$$

From [5], we have

$$
\|p\|_{L^{2}(\mathcal{M})}^{4} \leq C\left(\frac{1}{|\Im \lambda|}+|\Im \lambda|\left\|\Lambda_{q-\lambda}-\Lambda_{\tilde{q}-\lambda}\right\|_{\mathscr{B}\left(L^{2}(\partial \mathcal{M})\right)}\right), \quad \lambda \in \mathbb{C} \backslash \mathbb{R},
$$

where the constant $C$ only depends on $n, \mathcal{M}$ and $\aleph$.

Now if, for some fixed integer $\ell \geq 1,\left(\lambda_{k}, \psi_{k}\right)=\left(\tilde{\lambda}_{k}, \tilde{\psi}_{k}\right), k \geq \ell$, then the calculations in the preceding section yield

$$
\left\|\Lambda_{q-\lambda}-\Lambda_{\tilde{q}-\lambda}\right\|_{\mathscr{B}\left(L^{2}(\partial \mathcal{M})\right)} \leq C\left(\sum_{k=1}^{\ell} \frac{\lambda_{k}^{2}}{\left|\lambda_{k}-\lambda\right|}+\sum_{k=1}^{\ell} \frac{\tilde{\lambda}_{k}^{2}}{\left|\tilde{\lambda}_{k}-\lambda\right|}\right) .
$$

Again, the constant $C$ only depends on $n, \mathcal{M}$ and $\aleph$.

We deduce by combining together (3.1) and (3.2) that there exist two constants $C>0$ and $\kappa>0$, only depending on $n, \mathcal{M}, \aleph$ and $\ell$, so that

$$
\|p\|_{L^{2}(\mathcal{M})}^{4} \leq C\left(\frac{1}{|\Im \lambda|}+\frac{|\Im \lambda|}{|\lambda|}\right), \quad \lambda \in \mathbb{C} \backslash \mathbb{R},|\lambda| \geq \kappa
$$

We find by taking in this inequality $\lambda=(\tau+i)^{2}=\left(\tau^{2}-1\right)+2 i \tau$, with $\tau \geq 1$ sufficiently large,

$$
\|p\|_{L^{2}(\mathcal{M})}^{4} \leq C\left(\frac{1}{\tau}+\frac{\tau}{\tau^{2}-1}\right)
$$


Taking the limit, as $\tau \rightarrow \infty$, in this inequality, we obtain $p=0$. In particular, we proved that the spectral boundary data $\left(\lambda_{k}, \psi_{k}\right)_{k \geq \ell}$ determine uniquely $q \in$ $L^{\infty}(\mathcal{M}, \mathbb{R}) \cap H_{0}^{1}(\mathcal{M})$, for any arbitrary fixed integer $\ell \geq 1$ :

Theorem 3.1. Let $\ell \geq 1$ be an integer and assume that $\mathcal{M}$ is simple. If $q, \tilde{q} \in$ $L^{\infty}(\mathcal{M}, \mathbb{R}) \cap H_{0}^{1}(\mathcal{M})$ satisfy

$$
\lambda_{k}(q)=\lambda_{k}(\tilde{q}) \quad \text { and } \quad \psi_{k}(q)=\psi_{k}(\tilde{q}), \quad k \geq \ell,
$$

then $q=\tilde{q}$.

\section{REFERENCES}

[1] G. Alessandrini and J. Sylvester, Stability for multidimensional inverse spectral problem, Commun. Partial Diffent. Equat. 15 (5) (1990), 711-736. 3

[2] M. Belishev, An approach to multidimensional inverse problems for the wave equation, Dokl. Akad. Nauk SSSR 297 (1987), 524-527. 3

[3] M. Belishev and Y. Kurylev, To the reconstruction of a Riemannian manifold via its spectral data (BC-method), Commun. Partial Diffent. Equat. 17 (1992), 767-804. 3

[4] M. Bellassoued, M. Choulli, D. Dos Santos Ferreira, Y. Kian and P. Stefanov, A Borg-Levinson theorem for magnetic Schrödinger operators on a Riemannian manifold, arXiv: 1807.08857 .3

[5] M. Bellassoued, M. Choulli, Y. Kian and E. Soccorsi, Borg Levinson type theorem with partial spectral boundary data, preprint. 5

[6] M. Bellassoued, M. Choulli and M. Yamamoto, Stability estimate for an inverse wave equation and a multidimensional Borg-Levinson theorem, J. Diffent. Equat. 247 (2) (2009), 465-494. 3

[7] M. Bellassoued, M. Choulli and M. Yamamoto, Stability estimate for a multidimensional inverse spectral problem with partial data, J. Math. Anal. Appl. 378 (1) (2011), 184-197. 3

[8] P. Caro and M. Salo, Stability of the Calderón problem in admissible geometries, Inverse Problems and Imaging 8 (2014), 939-957. 2

[9] M. Choulli, Une introduction aux problèmes inverses elliptiques et paraboliques, Mathématiques et Applications, Vol. 65, Springer-Verlag, Berlin, 2009. 3

[10] M. Choulli and P. Stefanov, Stability for the multi-dimensional Borg-Levinson theorem with partial spectral data, Commun. Partial Diffent. Equat. 38 (3) (2013), 455-476. 3

[11] Oleg Yu. Imanuvilov and M. Yamamoto, Stability of determination of Riemannian metrics by spectral data and Dirichlet-to-Neumann map limited on arbitrary subboundary, Inverse Problems and Imaging 13 (6) (2019), 1213-1258. 3

[12] H. Isozaki, Some remarks on the multi-dimensional Borg-Levinson theorem, J. Math. Kyoto Univ. 31 (3) (1991), 743-753. 3

[13] A. Katchalov and Y. Kurylev, Multidimensional inverse problem with incomplete boundary spectral data, Commun. Partial Diffent. Equat. 23 (1998), 55-95. 3

[14] A. Katchalov, Y. Kurylev, M. Lassas, Inverse boundary spectral problems, Chapman \& Hall/CRC, Boca Raton, FL, 2001, 123, xx+290. 3

[15] O. Kavian, Y. Kian, E. Soccorsi, Uniqueness and stability results for an inverse spectral problem in a periodic waveguide, J. Math. Pures Appl. 104 (2015), no. 6, 1160-1189. 3

[16] Y. Kian, A multidimensional Borg-Levinson theorem for magnetic Schrödinger operators with partial spectral data, J. Spectral Theory 8 (2018), 235-269. 3

[17] Y. Kian, L. Oksanen, M. Morancey, Application of the boundary control method to partial data Borg-Levinson inverse spectral problem, Math. Control and Related Fields 9 (2019), 289-312. 3

[18] A. Nachman, J. Sylvester, G. Uhlmann, An n-dimensional Borg-Levinson theorem, Commun. Math. Phys. 115 (4) (1988), 595-605. 3

[19] V. Pohjola, Multidimensional Borg-Levinson theorems for unbounded potentials Asymptot. Anal. 110 (3-4) (2018), 203-226. 3

Université de Lorraine, 34 Cours Léopold, 54052 NAnCy Cedex, France

E-mail address: mourad.choulli@univ-lorraine.fr 